\title{
Simplified stock markets described by number operators
}

\author{
F. Bagarello \\ Dipartimento di Metodi e Modelli Matematici, Facoltà di Ingegneria, \\ Università di Palermo, Viale delle Scienze, I - 90128 Palermo, Italy \\ E-mail: bagarell@unipa.it \\ home page: www.unipa.it $\$ bagarell
}

\begin{abstract}
In this paper we continue our systematic analysis of the operatorial approach previously proposed in an economical context and we discuss a mixed toy model of a simplified stock market, i.e. a model in which the price of the shares is given as an input. We deduce the time evolution of the portfolio of the various traders of the market, as well as of other observable quantities. As in a previous paper, we solve the equations of motion by means of a fixed point like approximation.
\end{abstract}

Keywords: Stock markets; Canonical commutation relations. 


\section{Introduction and motivations}

In some recent papers, 11, 2, we have discussed why and how a quantum mechanical framework, and in particular operator algebras and the number representation, can be used in the analysis of some simplified models of stock markets. In these models, among the other simplifications, no financial derivative are considered at all. For this reason our interest is different from that discussed in [3], even if the frameworks appear to be very close. The main reason for using operator algebras in the analysis of this simplified closed stock market comes from the following considerations: in the closed market we have in mind the total amount of cash stays constant. Also, the total number of shares does not change with time. Moreover, when a trader $\tau$ interacts with a second trader $\sigma$, they change money and shares in a discrete fashion: for instance, $\tau$ increments his shares of 1 unit while his cash decrements of a certain number of monetary units (which is the minimum amount of cash existing in the market: 1 cent of dollar, for example), which is exactly the price of the share. Of course, for the trader $\sigma$ the situation is just reversed. So we have at least two quantities, the cash and the number of shares, which change as multiples of two fixed quantities. We further have two other quantities in our simplified market: the price of the share (in our simplified market the traders can exchange just a single kind of shares!) and the market supply, i.e. the overall tendency of the market to sell a share. For the same reason just discussed it is clear that also the price of the share must change discontinuously. It is therefore convenient to assume that also the market supply is labeled by a discrete quantity. In our naive version of a stock market the price of the share is related to the market supply in a very direct way: if the market supply increases, then the value of the share must decrease and viceversa. These are all the ingredients of our toy model of a stock market.

Operator algebras and quantum mechanics produce a very natural settings for discussing such a system. Indeed they produce a natural way for: (a) describing quantities which change with discrete steps; (b) obtaining the differential equations for the relevant variables of the system under consideration, the so-called observables of the system; (c) finding conserved quantities. It is well known indeed that operators satisfying the canonical commutation relations can be used quite naturally to discuss point (a) above. Hence the use of the Heisemberg dynamics, which is somehow intrinsic in quantum mechanics, 
appears a natural (but not exclusive!) choice to discuss points (b) and (c).

For these reasons we have suggested in [1, 2] an operator-valued scheme for the description of such a simplified market. We refer to the Appendix for some preliminary result.

It should be mentioned that our idea is not entirely new. Indeed, the use of quantum mechanical tools, and more in general of standard statistical techniques in the analysis of stock markets was already proposed by many authors, see for instance [3, 4, ,5, 6] and references therein. However, the approach discussed here and in [1, 2] is the first one, in our knowledge, in which the mechanism of the exchange between traders is given in terms of a boson-like based hamiltonian operator. This is close but somehow different from what is discussed in [3], where a quantum hamiltonian $H_{B}$ is introduced in the analysis of financial derivatives which is undertaken by looking at the Schrödinger equation arising from $H_{B}$.

It should also be mentioned that we are still very far from being able to deal with a real stock market, and this is the reason why our analysis still misses of a detailed comparison with existing methods, which will be undertaken on some more realistic model.

The paper is organized as follows:

In Section II we introduce the model originally proposed in [2], and we deduce the related equations of motion.

In Section III we modify this model by assuming different behaviors for the price of the share considered into the differential equations as an independent input. Then we discuss how the value of the portfolio of a given trader changes with time, analyzing some numerical results while our conclusions are given in Section IV. To keep the paper selfcontained we have included in Appendix few useful and standard results on the number representation.

\section{The all-in-one model}

In [2] we have introduced the following hamiltonian to describe the time evolution of some observables of a toy model of stock market consisting in $L$ traders exchanging shares of a single type, whose price is decided by the market itself, see below, 


$$
\left\{\begin{array}{l}
H=H_{0}+\lambda H_{I}, \text { where } \\
H_{0}=\omega_{a} \hat{n}+\omega_{c} \hat{k}+\omega_{p} \hat{P}+\sum_{k \in \Lambda}\left(\Omega_{A}(k) \hat{N}_{k}+\Omega_{C}(k) \hat{K}_{k}+\Omega_{O}(k) \hat{O}_{k}\right) \\
H_{I}=\left(z^{\dagger} Z(f)+z Z^{\dagger}(\bar{f})\right)+\left(p^{\dagger} o(g)+p o^{\dagger}(\bar{g})\right)
\end{array}\right.
$$

Here $\omega_{a}, \omega_{c}$ and $\omega_{p}$ are positive real numbers and $\Omega_{A}(k), \Omega_{C}(k)$ and $\Omega_{O}(k)$ are real valued positive functions. They define the free time evolution of the different operators of the market and $\lambda$ is a parameter measuring the strenght of the interactions between the different traders. Moreover, introducing the operators $a, c, p, o_{j}, A_{j}, C_{j}, j \in \Lambda$, and their adjoints, which are assumed to satisfy the following commutation rules

$$
\left[c, c^{\dagger}\right]=\left[p, p^{\dagger}\right]=\left[a, a^{\dagger}\right]=\mathbb{1}, \quad\left[o_{i}, o_{j}^{\dagger}\right]=\left[A_{i}, A_{j}^{\dagger}\right]=\left[C_{i}, C_{j}^{\dagger}\right]=\delta_{i, j} \mathbb{1},
$$

the operators appearing in (2.1) are defined as $\hat{n}=a^{\dagger} a, \hat{k}=c^{\dagger} c, \hat{P}=p^{\dagger} p, \hat{N}_{k}=A_{k}^{\dagger} A_{k}$, $\hat{K}_{k}=C_{k}^{\dagger} C_{k}$ and $\hat{O}_{k}=o_{k}^{\dagger} o_{k}$. In $H$ we have also introduced the following smeared fields, depending on two regular functions $f(k)$ and $g(k)$,

$$
\left\{\begin{array}{l}
Z(f)=\sum_{k \in \Lambda} Z_{k} f(k)=\sum_{k \in \Lambda} A_{k} C_{k}^{\dagger} \hat{P} f(k), \\
Z^{\dagger}(\bar{f})=\sum_{k \in \Lambda} Z_{k}^{\dagger} \overline{f(k)}=\sum_{k \in \Lambda} A_{k}^{\dagger} C_{k}^{\hat{P}} \overline{f(k)} \\
o(g)=\sum_{k \in \Lambda} o_{k} g(k) \\
o^{\dagger}(\bar{g})=\sum_{k \in \Lambda} o_{k}^{\dagger} \overline{g(k)}
\end{array}\right.
$$

as well as the operators $z=a c^{\dagger} \hat{P}, Z_{k}=A_{k} C_{k}^{\dagger^{\hat{P}}}$ and their conjugates. Notice that we have separated here our stock market into two main components: the single trader $\tau$, which is arbitrary but fixed, and all the other traders, $\left\{\sigma_{k}\right\}$, whose set we call $\mathcal{R}$. We refer to Table 1 below for a list of these operators and of their economical meaning, which can be deduced by the discussion following equation (A.4). 


\begin{tabular}{|c|c|}
\hline the operator and.. & ...its economical meaning \\
\hline$a$ & annihilates a share in the portfolio of $\tau$ \\
\hline$a^{\dagger}$ & creates a share in the portfolio of $\tau$ \\
\hline$\hat{n}=a^{\dagger} a$ & counts the number of shares in the portfolio of $\tau$ \\
\hline$c$ & annihilates a monetary unit in the portfolio of $\tau$ \\
\hline$c^{\dagger}$ & creates a monetary unit in the portfolio of $\tau$ \\
\hline$\hat{k}=c^{\dagger} c$ & counts the number of monetary units in the portfolio of $\tau$ \\
\hline$p$ & it lowers the price of the share of one unit of cash \\
\hline$p^{\dagger}$ & it increases the price of the share of one unit of cash \\
\hline$\hat{P}=p^{\dagger} p$ & gives the value of the share \\
\hline$A_{k}$ & annihilates a share in the portfolio of $\sigma_{k}$ \\
\hline$A_{k}^{\dagger}$ & creates a share in the portfolio of $\sigma_{k}$ \\
\hline$\hat{N}_{k}=A_{k}^{\dagger} A_{k}$ & counts the number of shares in the portfolio of $\sigma_{k}$ \\
\hline$C_{k}$ & annihilates a monetary unit in the portfolio of $\sigma_{k}$ \\
\hline$C_{k}^{\dagger}$ & creates a monetary unit in the portfolio of $\sigma_{k}$ \\
\hline$\hat{K}_{k}=C_{k}^{\dagger} C_{k}$ & counts the number of monetary units in the portfolio of $\sigma_{k}$ \\
\hline$o_{k}$ & "it lowers the k-th component of the market supply of one unit \\
\hline$o_{k}^{\dagger}$ & it increases the k-th component of the market supply of one unit \\
\hline$\hat{O}_{k}=o_{k}^{\dagger} o_{k}$ & gives the value of the the $\mathrm{k}$-th component of the market supply \\
\hline
\end{tabular}

Table 1.- List of operators and of their economical meaning.

Notice now that, because of (2.2), the following commutators can also be deduced, [2]:

$$
\left[\hat{K}_{k}, C_{q}^{\hat{P}}\right]=-\hat{P} C_{q}^{\hat{P}} \delta_{k, q}, \quad\left[\hat{K}_{k}, C_{q}^{\dagger} \hat{P}\right]=\hat{P} C_{q}^{\dagger} \hat{P} \delta_{k, q}
$$

To Table 1 we add now for completeness the economical meaning of, e.g., the operator $z$. This is rather evident, indeed. Let us now consider a fixed number vector, extending the one in (A.4),

$$
\varphi_{n, k, M,\{N\} ;\{K\} ;\{O\}}:=\frac{a^{\dagger^{n}} c^{\dagger k} p^{\dagger^{M}} A_{1}^{\dagger N_{1}} \cdots A_{L}^{\dagger N_{L}} C_{1}^{\dagger K_{1}} \cdots C_{L}^{\dagger K_{L}} o_{1}^{\dagger O_{1}} \cdots o_{L}^{\dagger O_{L}}}{\sqrt{n ! k ! M ! N_{1} ! \ldots N_{L} ! K_{1} ! \ldots K_{L} ! O_{1} ! \ldots O_{L} !}} \varphi_{0}
$$


where $\{N\}=N_{1}, N_{2}, \ldots, N_{L},\{K\}=K_{1}, K_{2}, \ldots, K_{L},\{O\}=O_{1}, O_{2}, \ldots, O_{L}$ and $\varphi_{0}$ is the vacuum of all the annihilation operators involved here.

The action of $z$ on such a vector destroys a share in the portfolio of the trader $\tau$ because of the presence of the annihilation operator $a$ and, at the same time, creates as many monetary units as $\hat{P}$ prescribes because of $c^{\dagger}{ }^{\hat{P}}$. Of course, in the interaction hamiltonian $H_{I}$ such an operator is associated to $Z^{\dagger}(\bar{f})$ which acts exactly in the opposite way on the other traders $\left\{\sigma_{k}\right\}$ of the market: one share is created in the cumulative portfolio of $\mathcal{R}$ while $\hat{P}$ quanta of money are destroyed, since they are used to pay for the share. This interpretation clearly follows from the commutation rules in (2.2) and (2.4). That's why $z$ is called a selling and $Z^{\dagger}(\bar{f})$ a buying operator.

Finally, if $L+1$ is the total number of traders of our market, then the cardinality of $\Lambda$, which is a subset of $\mathbb{N}$ which labels the traders of $\mathcal{R}$, is obviously $L$.

The main aim of our analysis is to recover the equations of motion for the portfolio of the trader $\tau$ defined as

$$
\hat{\Pi}(t)=\hat{P}(t) \hat{n}(t)+\hat{k}(t) .
$$

This is a natural definition, since it is just the sum of the cash and of the total value of the shares that $\tau$ possesses at time $t$. Once again, we stress that in our simplified model no room is given to the financial derivatives, and not even to any different and realistic mechanism but a simple exchange cash $\leftrightarrow$ shares. This oversimplification makes our model reasonably simple to be analyzed using standard perturbative techniques but, at the same time, strongly limits the validity of the model itself. However, to our knowledge, this is a feature shared by most of the models of stock markets existing in the literature, which are usually focused just on some particular aspect of the market and not, because of its complexity, to the whole system.

Remarks:- (1) Table 1 has a physical origin which is discussed in [2] where the socalled stochastic limit approach for quantum open systems was adopted. The role of the smeared field in this context has been clarified. It may be worth stressing that what we call stochastic limit approach is essentially a perturbative technique originally discussed in a quantum mechanical context, [7].

(2) Of course, since $\tau$ can be chosen arbitrarily, the asymmetry of the model is just apparent. In fact, changing $\tau$, we will be able, in principle, to find the time evolution of 
the portfolio of each trader of the stock market.

The interpretation suggested above concerning $z$ and $Z(f)$ is also based on the following result: let us define the operators

$$
\hat{N}:=\hat{n}+\sum_{k \in \Lambda} \hat{N}_{k}, \quad \hat{K}:=\hat{k}+\sum_{k \in \Lambda} \hat{K}_{k}, \quad \hat{\Gamma}:=\hat{P}+\sum_{k \in \Lambda} \hat{O}_{k}
$$

We have seen in [2] that $\hat{N}, \hat{K}$ and $\hat{\Gamma}$ are constants of motion: $[H, \hat{N}]=[H, \hat{K}]=[H, \hat{\Gamma}]=$ 0 . This is in agreement with our interpretation: we are constructing a closed market in which the total amount of money and the total number of shares are preserved and in which, if the total supply increases, then the price of the share must decrease in such a way that $\hat{\Gamma}$ stays constant.

In 2] we have shown how to use the stochastic limit approach or a fixed point-like (fpl) procedure to get suitable approximations of $\hat{\Pi}(t)$ in (2.6). Here we just consider this last approach, since it is more relevant for the analysis we will perform here. We refer to [2] for the details of our derivation, and for the details on the assumptions that have been taken along the way. Here we just write down the system of operatorial differential equations which we have deduced and which looks like

$$
\left\{\begin{array}{l}
\frac{d \hat{n}(t)}{d t}=i \lambda\left(-z^{\dagger}(t) Z(f, t)+z(t) Z^{\dagger}(\bar{f}, t)\right) \\
\frac{d \hat{k}(t)}{d t}=i \lambda P_{c}(t)\left(z^{\dagger}(t) Z(f, t)-z(t) Z^{\dagger}(\bar{f}, t)\right) \\
\frac{d z(t)}{d t}=i\left(P_{c}(t) \omega_{c}-\omega_{a}\right) z(t)+i \lambda\left[z^{\dagger}(t), z(t)\right] Z(f, t) \\
\frac{d Z(f, t)}{d t}=i Z\left(\left(P_{c}(t) \Omega_{C}-\Omega_{A}\right) f, t\right)+i \lambda z(t)\left[Z^{\dagger}(\bar{f}, t), Z(f, t)\right]
\end{array}\right.
$$

where

$$
P_{c}(t)=\frac{1}{2}[(M+O)+(M-O) \cos (2 \lambda t)]
$$

is the mean value of the operator $\hat{P}(t)$ on a vector state defined by (2.5), and which we simply indicate with $\omega$, and can be explicitly found due to the simple expression of $H$, [2]: $P_{c}(t)=\omega(\hat{P}(t))$. Here $M$ and $O$ are respectively the initial price of the shares and the initial value of the market supply (which is related to the set $\{O\}$ in (2.5)). These equations produce the dynamical behavior of our market after taking their mean value on the number vector state $\omega,[2$. As mentioned in Appendix, this state has a double effect: first of all, it allows us to move from the quantum dynamics of the model to its 
classical counterpart, since we use $\omega$ to replace the time dependent operators with their mean values, which are ordinary functions of time. Secondly, such a vector state is used to fix the initial conditions of the differential equations, that is the initial number of shares, the initial cash and so on.

In order to simplify the analysis of this system we have assumed in [2] that both $\Omega_{C}(k)$ and $\Omega_{A}(k)$ are constant for $k \in \Lambda$. Indeed, under this assumption, the last two equations in (2.8) form by themselves a closed system of differential equations in the non abelian variables $z(t)$ and $Z(f, t)$ :

$$
\left\{\begin{array}{l}
\frac{d z(t)}{d t}=i\left(P_{c}(t) \omega_{c}-\omega_{a}\right) z(t)+i \lambda Z(f, t)\left[z^{\dagger}(t), z(t)\right] \\
\frac{d Z(f, t)}{d t}=i\left(P_{c}(t) \Omega_{C}-\Omega_{A}\right) Z(f, t)+i \lambda z(t)\left[Z^{\dagger}(\bar{f}, t), Z(f, t)\right] .
\end{array}\right.
$$

Getting the exact solution of the system (2.8), with (2.10) replacing the two last equations, is a hard job. However, a natural approximation can be constructed considering the fpl approach for which, again, we refer to [2]. It should be mentioned, however, that for the time being we have not undertaken the rigorous analysis of such an approximation (i.e. the estimate of the error), which is adopted here mainly because it can be very easily implemented and, more than this, because it is quite natural. The result is the following: $z(t)$ and $Z(f, t)$ can be approximated by two different (and not commuting) operators $z_{1}(t)$ and $Z_{1}(f, t),[2]$ :

$$
z_{1}(t)=z \eta_{1}(t)+Z(f)\left[z^{\dagger}, z\right] \eta_{2}(t), \quad Z_{1}(f, t)=Z(f) \tilde{\eta}_{1}(t)+z\left[Z(\bar{f})^{\dagger}, Z(f)\right] \tilde{\eta}_{2}(t)
$$

where

$$
\left\{\begin{array}{l}
\eta_{1}(t)=1+i \int_{0}^{t}\left(P_{c}\left(t^{\prime}\right) \omega_{c}-\omega_{a}\right) e^{i \chi\left(t^{\prime}\right)} d t^{\prime}, \quad \eta_{2}(t)=i \lambda \int_{0}^{t} e^{i \tilde{\chi}\left(t^{\prime}\right)} d t^{\prime} \\
\tilde{\eta}_{1}(t)=1+i \int_{0}^{t}\left(P_{c}\left(t^{\prime}\right) \Omega_{C}-\Omega_{A}\right) e^{i \tilde{\chi}\left(t^{\prime}\right)} d t^{\prime}, \quad \tilde{\eta}_{2}(t)=i \lambda \int_{0}^{t} e^{i \chi\left(t^{\prime}\right)} d t^{\prime}
\end{array}\right.
$$

and $\chi(t)=\alpha t+\beta \sin (2 \lambda t), \tilde{\chi}(t)=\tilde{\alpha} t+\tilde{\beta} \sin (2 \lambda t)$, where $\alpha=\frac{1}{2}\left((M+O) \omega_{c}-2 \omega_{a}\right)$, $\tilde{\alpha}=\frac{1}{2}\left((M+O) \Omega_{C}-2 \Omega_{A}\right), \beta=\frac{\omega_{c}}{4 \lambda}(M-O), \tilde{\beta}=\frac{\Omega_{C}}{4 \lambda}(M-O)$.

It is now easy to find that the mean values of the first two equations in (2.8) on the state $\omega$ can be written as

$$
\left\{\begin{array}{l}
\dot{n}(t)=\frac{d n(t)}{d t}=-2 \lambda \Im\left\{\omega\left(z(t) Z^{\dagger}(\bar{f}, t)\right)\right\}, \\
\dot{k}(t)=\frac{d k(t)}{d t}=2 \lambda P_{c}(t) \Im\left\{\omega\left(z(t) Z^{\dagger}(\bar{f}, t)\right)\right\},
\end{array}\right.
$$


which in particular implies that $P_{c}(t) \dot{n}(t)+\dot{k}(t)=0$ for all $t$. Hence we find that $\dot{\Pi}(t)=$ $\dot{P}_{c}(t) n(t)$. It should be remarked that, because of this relation, since $M=O$ in (2.9) implies $P_{c}(t)=P_{c}(0)=M$, then when $M=O$ the dynamics of the portfolio of $\tau$ is trivial, $\Pi(t)=\Pi(0)$, even if both $n(t)$ and $k(t)$ may change in time.

Let us now insert $z_{1}(t)$ and $Z_{1}(f, t)$ in equations (2.13). If $\omega$ is the usual number state, and if we call

$$
\left\{\begin{array}{l}
\omega(1):=\omega\left(z z^{\dagger}\left[Z^{\dagger}(\bar{f}), Z(f)\right]\right\} \\
\omega(2):=\omega\left(Z(f) Z^{\dagger}(\bar{f})\left[z^{\dagger}, z\right]\right\} \\
r(t)=\omega(1) \eta_{1}(t) \overline{\tilde{\eta}_{2}(t)}+\omega(2) \eta_{2}(t) \overline{\tilde{\eta}_{1}(t)}
\end{array}\right.
$$

then we get

$$
\left\{\begin{array}{l}
n(t)=n-2 \lambda \Im\left\{\int_{0}^{t} r\left(t^{\prime}\right) d t^{\prime}\right\}, \\
k(t)=k+2 \lambda \Im\left\{\int_{0}^{t} P_{c}\left(t^{\prime}\right) r\left(t^{\prime}\right) d t^{\prime}\right\} .
\end{array}\right.
$$

The time dependence of the portfolio can now be written as

$$
\Pi(t)=\Pi(0)+\delta \Pi(t)
$$

with

$$
\begin{gathered}
\delta \Pi(t)=n(O-M) \sin ^{2}(\lambda t)+ \\
+\left(-2 \lambda \Im\left\{\int_{0}^{t} r\left(t^{\prime}\right) d t^{\prime}\right\}\left(M+(O-M) \sin ^{2}(\lambda t)\right)+2 \lambda \Im\left\{\int_{0}^{t} P_{c}\left(t^{\prime}\right) r\left(t^{\prime}\right) d t^{\prime}\right\}\right),
\end{gathered}
$$

which gives the variation of the portfolio of $\tau$ in time.

Remark: it is worth noticing that $\delta \Pi(t)=0$ for all $t$ if $\lambda=0$. This is reasonable, since $\lambda=0$ means no interaction in (2.1) and, as a consequence, no way to change the original status quo.

In [2] we have discussed some particular solutions of this system under special conditions. In particular we have shown that there exist situations which are rather convenient for the trader $\tau$, meaning with this that $\delta \Pi(t) \geq 0$ for all $t \geq 0$ : under these conditions, within our framework and our approximations, the total value of the portfolio of $\tau$ never decreases! These conditions are, not surprisingly, related to the constants which enter in the definition of $H$. However, due to the simplified mechanism which fixes the price of the share, it is clear that we cannot trust very much these conclusions: as already stated, the 
model still needs further improvements. In particular different kind of shares and a more realistic dynamics for the price must still be implemented. Both these extensions require some care, and will be considered in a series of papers which are now in preparation. We consider a first generalization in the next section, which is used to get a deeper insight on the role of the constants appearing in the definition of the model itself.

\section{The mixed model}

In this section we discuss in some details a different model of a stock market, based again on almost all the same assumptions introduced in [1, 2]. We call this a mixed model since it has an hamiltonian ingredient, which involves again the cash and the number of shares of both $\tau$ and of the traders in $\mathcal{R}$, and an empirical part, since the dynamics of the price is not deduced as before from the market supply but simply given as an input in (2.8), which is here replaced by the following system

$$
\left\{\begin{array}{l}
\frac{d \hat{n}(t)}{d t}=i \lambda\left(-z^{\dagger}(t) Z(f, t)+z(t) Z^{\dagger}(\bar{f}, t)\right), \\
\frac{d \hat{k}(t)}{d t}=i \lambda P(t)\left(z^{\dagger}(t) Z(f, t)-z(t) Z^{\dagger}(\bar{f}, t)\right), \\
\frac{d z(t)}{d t}=i\left(P(t) \omega_{c}-\omega_{a}\right) z(t)+i \lambda Z(f, t)\left[z^{\dagger}(t), z(t)\right], \\
\frac{d Z(f, t)}{d t}=i\left(P(t) \Omega_{C}-\Omega_{A}\right) Z(f, t)+i \lambda z(t)\left[Z^{\dagger}(\bar{f}, t), Z(f, t)\right] .
\end{array}\right.
$$

Hence here $P(t)$ is a classical external field, for which we will consider different analytical expressions in the rest of the paper.

The main reason why we are considering this simplified model is that the models proposed in [1, 2] and in the previous section, all depend on a set of parameters whose role in the description of our toy stock market needs to be yet fully understood. At a preliminary stage it is therefore convenient to reduce the number of degrees of freedom and to deal with the simpler model described by (3.1). This should produce a deeper understanding which will be useful to construct other and more realistic models. This analysis is also necessary if we want to compare our analysis with the ones already existing in the literature, where the role of certain parameters is quite often absolutely crucial, see [8, 9] for instance. The relation between these parameters, for instance, decides the nature of the traders which form our market, [9], or how these traders react to the dynamics of the market. 
Needless to say, a realistic model of a stock market should contain, first of all, more than a single kind of share. Moreover, a mechanism which fixes the prices of all these shares is needed, and this is surely not a trivial task since interactions between the various traders and the various kind of shares must be taken into account, as well as external sources of information. This is exactly our final aim: produce a model which contains $N$ traders and $L$ different kind of shares whose prices are decided by some global mechanism to be identified. However, for the time being, we just continue our preliminary analysis, paying particular attention to the role of the parameters of the simplified model described here.

Remark:- One could think to deduce system (3.1) from the following effective (timedependent) hamiltonian $H_{\text {eff }}$ which looks very similar to that in (2.1)

$$
\left\{\begin{array}{l}
H_{e f f}(t)=H_{e f f, 0}+\lambda H_{e f f, I}(t), \text { where } \\
H_{e f f, 0}=\omega_{a} \hat{n}+\omega_{c} \hat{k}+\sum_{k \in \Lambda}\left(\Omega_{A} \hat{N}_{k}+\Omega_{C} \hat{K}_{k}\right) \\
\left.H_{e f f}(t)=\left(z^{\dagger}(t) Z(f, t)+z(t) Z^{\dagger}(\bar{f}, t)\right)\right)
\end{array}\right.
$$

where, for instance, $Z(f, t)=\sum_{k \in \Lambda} Z_{k} f(k)=\sum_{k \in \Lambda} f(k) A_{k} C_{k}^{\dagger}{ }^{(t)}$. However this is not rigorous because the time dependence of $P(t)$ modifies the standard Heisenberg equation $\frac{d}{d t} X(t)=i[H, X(t)]$. Hence it is more convenient to take (3.1) as the starting point, and (3.2) just as a formal hamiltonian heuristically related to the economical system.

As for the numerical aspects, we have restricted here the analysis of the model to a time interval $t \in\left[0, t_{0}\right]$, with $t_{0}=6$ just to fix the ideas, and we have considered the following forms for $P(t): P_{1}(t)=t$,

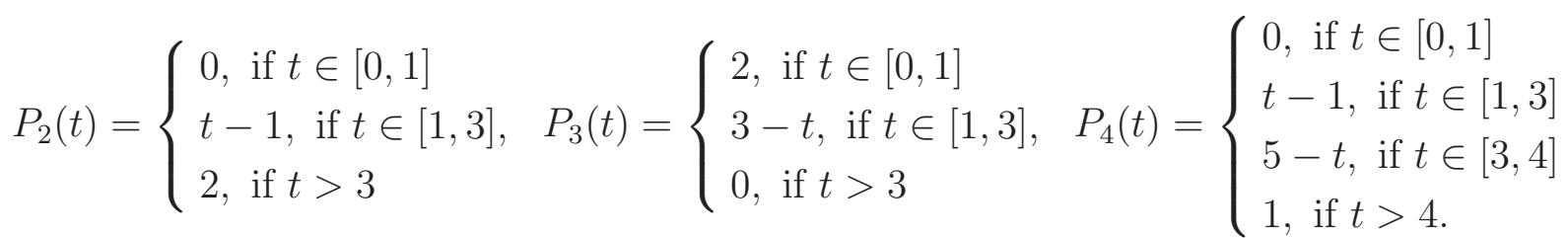

Hence, we are considering four different situations: $P_{1}(t)$ and $P_{2}(t)$ describe a not decreasing price, while $P_{3}(t)$ is a not increasing function. Finally, $P_{4}(t)$ describes a share whose value increases up to a maximum and then decreases and reaches a limiting value. 
The reason for using these rather than other and more regular functions is that with these choices it is easier to compute analytically some of the quantities appearing in the solution of system (3.1).

Let us now show quickly how to find this solution by means of the fpl approximation. For more details we refer to [2]. As in Section II the main idea is finding first an approximated solution of the last two equations of (3.1) and then using these solutions in the first two equations of the same system. In this way we recover the solution in (2.11)-(2.12). In particular this last equation can be rewritten as

$$
\begin{cases}\eta_{1}(t)=e^{i \chi(t)}, & \eta_{2}(t)=i \lambda \int_{0}^{t} e^{i \tilde{\chi}\left(t^{\prime}\right)} d t^{\prime} \\ \tilde{\eta}_{1}(t)=e^{i \tilde{\chi}(t)}, & \tilde{\eta}_{2}(t)=i \lambda \int_{0}^{t} e^{i \chi\left(t^{\prime}\right)} d t^{\prime}\end{cases}
$$

with $\chi(t)=\int_{0}^{t}\left(P\left(t^{\prime}\right) \omega_{c}-\omega_{a}\right) d t^{\prime}$ and $\tilde{\chi}(t)=\int_{0}^{t}\left(P\left(t^{\prime}\right) \Omega_{C}-\Omega_{A}\right) d t^{\prime}$. These results can be used in the first two equations in (3.1) and we get

$$
\left\{\begin{array}{l}
n(t)=n+\delta n(t)=n-2 \lambda \int_{0}^{t} \Im\left(r\left(t^{\prime}\right)\right) d t^{\prime} \\
k(t)=k+\delta k(t)=k+2 \lambda \int_{0}^{t} P\left(t^{\prime}\right) \Im\left(r\left(t^{\prime}\right)\right) d t^{\prime},
\end{array}\right.
$$

with obvious notation, having introduced the function $r(t)$ as in (2.14). The time evolution of the portfolio of $\tau, \Pi(t)=\Pi(0)+\delta \Pi(t)$, can be written as

$$
\delta \Pi(t)=n(P(t)-P(0))+P(t) \delta n(t)+\delta k(t)
$$

which reduces to (2.17) under the assumptions of Section II.

In the rest of the paper we consider some simplifying conditions, useful to display easily our results: first of all, as in [2], we reduce the reservoir of the market just to another trader, $\sigma$. This makes the computation of $\omega(1)$ and $\omega(2)$ in (2.14) rather easy and we get

$$
\left\{\begin{array}{l}
\omega(2)=-|f(1)|^{2}\left(1+n^{\prime}\right) \frac{k !}{(k-M) !} \frac{k^{\prime} !}{\left(k^{\prime}-M\right) !} \\
\omega(1)=\omega(2)+|f(1)|^{2} n^{\prime} \frac{k !}{(k-M) !} \frac{\left(k^{\prime}+M\right) !}{k^{\prime} !}
\end{array}\right.
$$

which depends, as expected, on the initial values of the market. As a second assumption, we just consider the case of a trader $\tau$ which is entering into the market, so that it possess no share at all at $t=0$. Therefore we take $n=0$. This has an immediate consequence: in order to have an economical meaning, $\delta n(t)$ can only be non negative! This is actually what happens in all the examples we have considered so far. 


\section{III.1 Numerical results and conclusions}

We discuss now the numerical results of the solutions of system (3.1) for the different choices of $P(t)$ introduced previously. Here, for concreteness' sake, we fix $f(1)=10^{-3}$ in (3.6) and, as already mentioned, $n=0$, and we consider the following cases (different choices of constants in $H$ ) and subcases (different initial conditions):

case I: $\left(\omega_{a}, \omega_{c}, \Omega_{A}, \Omega_{C}\right)=(1,1,1,1)$; case II: $\left(\omega_{a}, \omega_{c}, \Omega_{A}, \Omega_{C}\right)=(10,10,1,1)$; case III: $\left(\omega_{a}, \omega_{c}, \Omega_{A}, \Omega_{C}\right)=(1,1,10,10)$; case IV: $\left(\omega_{a}, \omega_{c}, \Omega_{A}, \Omega_{C}\right)=(20,10,5,1) ;$ case V: $\left(\omega_{a}, \omega_{c}, \Omega_{A}, \Omega_{C}\right)=(1,5,10,20)$; case VI: $\left(\omega_{a}, \omega_{c}, \Omega_{A}, \Omega_{C}\right)=(1,3,2,7)$.

Subcase a: $\left(k, k^{\prime}, n^{\prime}, M\right)=(20,20,100,2) ;$ subcase b: $\left(k, k^{\prime}, n^{\prime}, M\right)=(80,20,100,2)$; subcase c: $\left(k, k^{\prime}, n^{\prime}, M\right)=(20,80,100,2)$; subcase d: $\left(k, k^{\prime}, n^{\prime}, M\right)=(80,80,100,2)$.

The first interesting result of our numerical computations is that there are several situations, like for instance IIIa, Va and VIa for $P_{1}(t)$, or IIIa, IVa, Va and VIa for $P_{2}(t)$, for which the variation of $\delta n$ is larger than zero but always (i.e. for $t<6$ ) strictly smaller than 1. Analogously there are other situations in which $\delta k(t)$, belongs to the interval ] $-1,1$ [ for $t<6$, like in IIIa, Va and VIa for $P_{1}(t)$. This can be interpreted as follows: since there is not much cash going around the market, it is quite unlikely that some transaction may take place between the two traders $\tau$ and $\sigma$. Figure I show $\delta n(t)$ and $\delta k(t)$ for $P_{1}(t)$ in case Va.
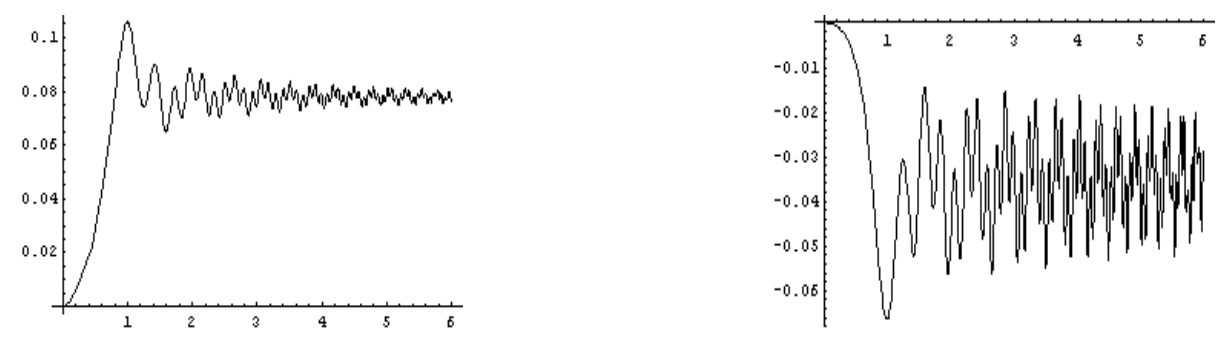

Figure 1: $\delta n(t)$ (left) and $\delta k(t)$ (right) for $P_{1}(t)$, case Va: no transaction is possible for $t \in[0,6]$

Moreover, there are situations in which the total quantity of cash or the number of shares exceed their initial values, like for instance in cases Id or IId for $P_{2}(t)$, see 
Figure 2 below. This is clearly incompatible with the fact that the total amount of cash and the total number of shares should be constant in time, and is uniquely due to the fpl approximation which we have adopted to solve the original system of differential equations. In other words, in these cases there exists a value of $t, t_{f}<6$, such that for $t>t_{f}$ the fpl approximation does not work anymore and our results become meaningless. This happens usually in each subcase $d$, because of the high values assumed by $\omega(1)$ and $\omega(2)$ due to the initial conditions. This problem could be solved if we adopt a better approximation to solve the differential equations in (3.1). This is part of the work in progress, but is far from being an easy task, due to the non-commutative nature of the system (3.1), which is also non linear. A more promising approach seems the use of timedependent perturbation theory rather than the fpl approximation considered here. This is also work in progress.
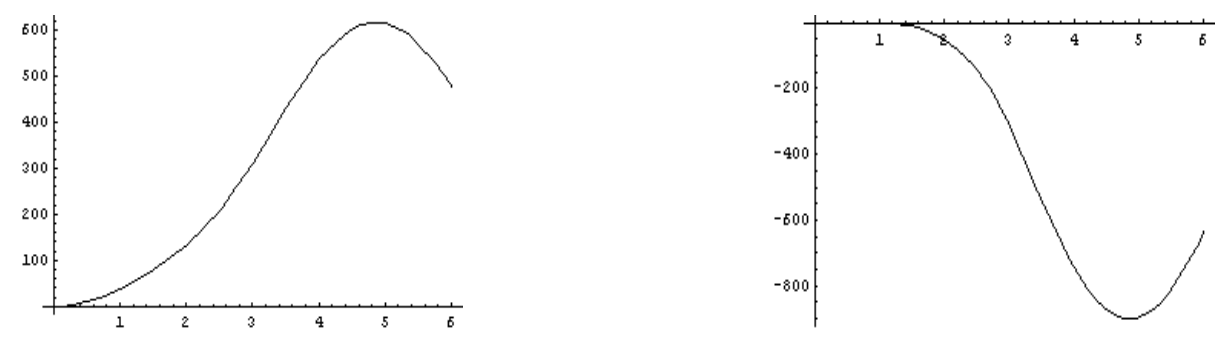

Figure 2: $\delta n(t)$, case Id (left) and $\delta k$, case IId (right), for $P_{2}(t)$ : the fpl approximation does not hold in all of $[0,6]$

As already mentioned, in all the $P(t)$ we have considered in this paper we have found that, for all $t$ in our range, $\delta n(t)$ is not negative, as it should since we are just considering the initial condition $n=0$ : hence the fpl approximation respects this feature of the model. We also observe that the range of variations of all the relevant observables, i.e. $\delta n(t), \delta k(t)$ and $\delta \Pi(t)$, is minimum in subcases a, maximum in the subcases d, while is intermediate for both subcases b and c: this is clearly due to the fact that different initial conditions imply different values of $\omega(1)$ and $\omega(2)$, which for instance increase very fast with $k$ and $k^{\prime}$, see (3.6). We further observe that the analytic behavior of $\delta \Pi(t)$ is close (sometimes very close) to that of $P(t)$. This is shown in Figure 3, which is obtained 
considering $P(t)=P_{4}(t)$ : in the upper figure we plot $P_{4}(t)$, while the three figures down shows $\delta \Pi(t)$ in case Ic (left), IVd (center) and Vc (right). From these figures it appears clearly that when the price of the share stays constant, there is no variation of $\delta \Pi(t)$. On the contrary, this increases when $P(t)$ increases and decreases when $P(t)$ decreases. This is not a peculiar feature of this example but can be analytically deduced from formula (3.5) with $n=0$. Indeed, if we just compute the time derivative of both side and use the definitions of $\delta n(t)$ and $\delta k(t)$, we find that $\dot{\Pi}(t)=\dot{P}(t) n(t)$ and, since $n(t)$ is non negative, $\dot{\Pi}(t)$ and $\dot{P}(t)$ have the same sign. Figure 3 also shows that there are conditions (Vc, for instance), in which $\delta \Pi(t)$ looks very much the same as $P(t)$, while with different choices of the constant of the hamiltonian and of the initial conditions the shape of $\delta \Pi(t)$ appear sufficiently different from that of $P(t)$. Of course this is an interesting feature of the model but is also a measure of how oversimplified the real system is here. A more realistic result could be found, maybe, introducing other mechanisms which are missing in our toy model. We will briefly come back to this point in the next section.
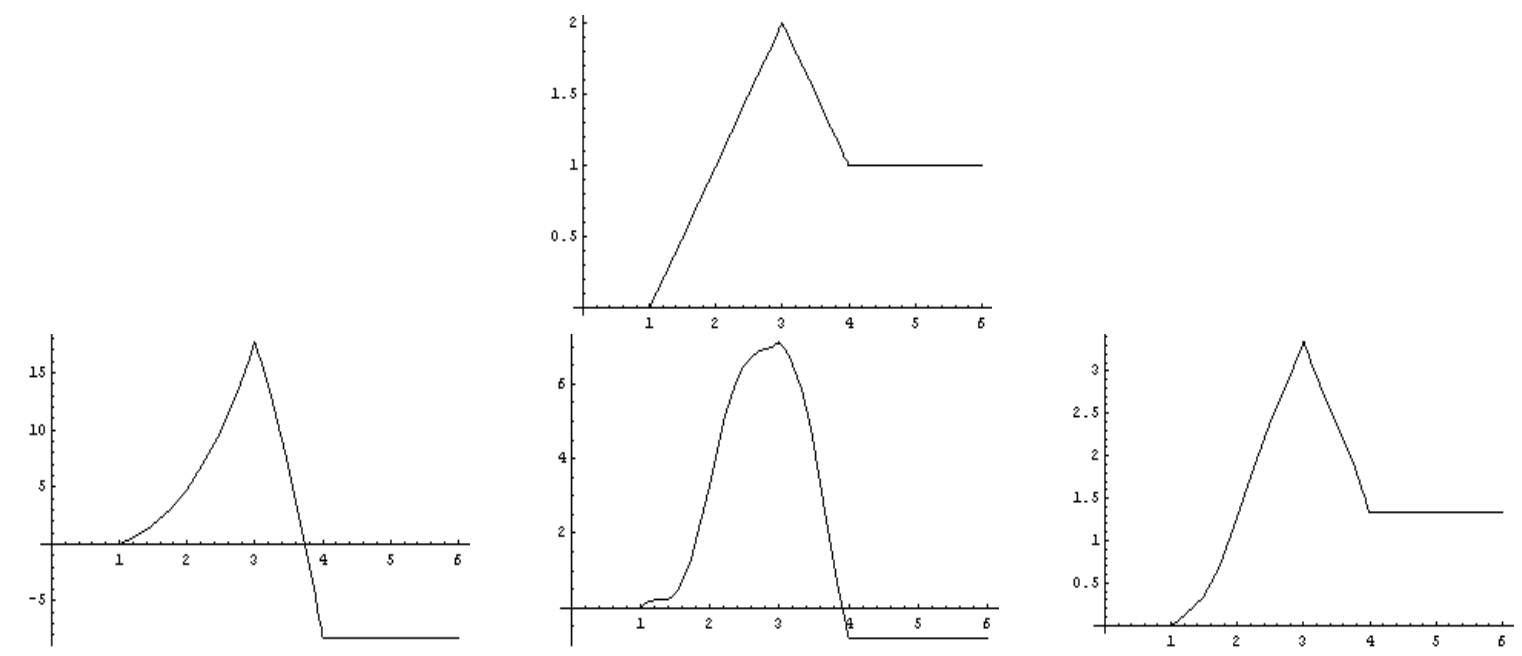

Figure 3: up: $P_{4}(t)$; down: $\delta \Pi(t)$ in cases Ic (left), IVd (middle), Vc (right)

We end this preliminary analysis by focusing our attention on the variation of the portfolio of $\tau$ : in the following table we show the range of variation of $\delta \Pi(t)$ for the 
different cases, subcases and choices of $P(t)$. Notice that the numbers are deduced from the plots, so that they are only indicative.

\begin{tabular}{|c|c|c|c|c|}
\hline & $P_{1}(t)$ & $P_{2}(t)$ & $P_{3}(t)$ & $P_{4}(t)$ \\
\hline I a & from 0 to 4 & from 0 to 1.2 & from -1.2 to 0 & from -0.5 to 1.25 \\
\hline I b & from 0 to 60 & from 0 to 17.5 & from -17.5 to 0 & from -7 to 17 \\
\hline I c & from 0 to 70 & from 0 to 20 & from -20 to 0 & from -8 to 20 \\
\hline I d & from 0 to 900 & from 0 to 300 & from -300 to 0 & from -110 to 300 \\
\hline II a & from 0 to 3.5 & from 0 to 1 & from -1 to 0 & from -0.4 to 1 \\
\hline II b & from 0 to 55 & from 0 to 17 & from -16 to 0 & from -7 to 17 \\
\hline II c & from 0 to 55 & from 0 to 17.5 & from -17.5 to 0 & from -7 to 17 \\
\hline II d & from 0 to 900 & from 0 to 270 & from -280 to 0 & from -100 to 250 \\
\hline III a & from 0 to 1 & from 0 to 0.35 & from -0.3 to 0 & from -0.1 to 0.3 \\
\hline III b & from 0 to 8 & from 0 to 2.5 & from -2.5 to 0 & from -0.5 to 2.5 \\
\hline III c & from 0 to 17.5 & from 0 to 5.2 & from -5.2 to 0 & from -2 to 5.3 \\
\hline III d & from 0 to 140 & from 0 to 43 & from -43 to 0 & from -10 to 45 \\
\hline$\overline{\text { IV a }}$ & from 0 to 0.7 & from 0 to 0.025 & from -0.15 to 0 & from -0.01 to 0.03 \\
\hline IV b & from 0 to 9 & from 0 to 0.4 & from -1.4 to 0 & from -0.05 to 0.4 \\
\hline IV c & from 0 to 10 & from 0 to 0.45 & from -2.5 to 0 & from -0.2 to 0.42 \\
\hline IV d & from 0 to 150 & from 0 to 7 & from -22 to 0 & from -0.4 to 7 \\
\hline$\overline{\mathrm{V} \text { a }}$ & from 0 to 0.4 & from 0 to 0.2 & from -0.03 to 0 & from 0 to 0.2 \\
\hline $\mathrm{V} \mathrm{b}$ & from 0 to 6 & from 0 to 1.25 & from -0.4 to 0 & from 0 to 1.3 \\
\hline $\mathrm{Vc}$ & from 0 to 7 & from 0 to 3.2 & from -0.5 to 0 & from 0 to 3.5 \\
\hline $\mathbf{V d}$ & from 0 to 100 & from 0 to 22 & from -7 to 0 & from 0 to 22 \\
\hline$\overline{\mathrm{VI}}$ & from 0 to 0.6 & from 0 to 0.7 & from -0.06 to 0 & from 0 to 0.7 \\
\hline $\mathrm{VI} \mathrm{b}$ & from 0 to 8 & from 0 to 9 & from -0.6 to 0 & from 0 to 9 \\
\hline VI c & from 0 to 10 & from 0 to 12 & from -1 to 0 & from 0 to 12 \\
\hline VI d & from 0 to 120 & from 0 to 150 & from -11 to 0 & from 0 to 150 \\
\hline
\end{tabular}


Table 2.- Range of variation of $\delta \Pi(t)$ for different choices of $P_{j}(t)$.

We see that for all given subcase, case $I$ is the one in which we find the widest range of variation of $\delta \Pi(t)$. In particular, for $P_{j}(t)$ with $j=1,2,4, \delta \Pi(t)$ increases its value more than in the other cases (II-VI) for any fixed subcase. If $j=3$ then $\delta \Pi(t)$ decreases its value in case I more than in the other cases (II-VI). This difference between $P_{3}(t)$ and the others $P_{j}(t), j \neq 3$, is clearly related to our previous remark concerning the signs of $\delta \dot{\Pi}(t)$ and $\dot{P}(t)$. If we now compare cases II and III we see that $\tau$ is favored for the first choice of constants more than for the second. Therefore we could interpret $\omega_{a}, \omega_{c}, \Omega_{A}$ and $\Omega_{C}$ as related to the information reaching respectively $\tau$ and $\sigma$ : when $\tau$ gets a larger amount of information he is able to earn more! However this is clearly only part of the conclusion, since also in case III the function $\delta \Pi(t)$ is positive, even if $\Omega_{A}=\Omega_{C}=10>1=\omega_{a}=\omega_{c}$, so that $\tau$ still increases the value of its portfolio. Moreover, if we consider the case IV in which $\omega_{a}$ and $\omega_{c}$ are even larger (and much larger than $\Omega_{A}$ and $\Omega_{C}$ ), we see from our table that $\tau$ does not improve his portfolio as in cases I, II and III: so what seems to be relevant is $\omega_{a}-\omega_{c}$ and $\Omega_{A}-\Omega_{C}$ more than the values of the constants themselves. However, this is not enough: our numerical results clearly show that there exists some asymmetry between $\left(\omega_{a}, \omega_{c}\right)$ and $\left(\Omega_{A}, \Omega_{C}\right)$ : the $\omega$ 's carry a higher amount of information than that of the $\Omega$ 's. This explains also the results in I. The reason for this asymmetry is not clear, at this moment, and further investigations will be undertaken soon, even if it could be simply related to the presence of $f(1)$, which only appears in $\mathcal{R}$ and not for $\tau$. A phenomenological law that could display these results, at least when $\omega_{a}=\omega_{c}=: \omega$ and $\Omega_{A}=\Omega_{C}=: \Omega$, is related to a sort of mean value of $\dot{P}(t)$ : if we put $\langle\dot{P}\rangle:=\frac{1}{T} \int_{0}^{T} \dot{P}(t) d t=\frac{P(T)-P(0)}{T}$, we see that $\delta \Pi(t)$ has the following expression:

$$
\delta \Pi(t)=\frac{\operatorname{sign}(\langle\dot{P}\rangle)}{g(\omega, \Omega)},
$$

where $g(\omega, \Omega)$ is a certain function which takes its minimum value for $\omega=\Omega$ and satisfies the inequality $g(\omega, \Omega)<g(\Omega, \omega)$ if $\omega>\Omega$. 


\section{Conclusions}

In this paper we have continued the analysis of stock markets began in [1, 2]. In particular, the main difference with respect to what we have done in [2] consists in the replacement of the simple mechanism which was considered in [2] to fix the price of the share with an external field, which is assumed here to be a known function of time. This is useful to get a deeper understanding of the various ingredients of our model, especially in view of further generalizations which should produce more realistic models, first introducing several kind of shares and then looking for a reasonable mechanism which fixes the price of the shares themselves. It is also necessary to improve the fpl-approximation we have adopted here, in order to avoid the spurious non-conservation of the integrals of motion observed in our numerical results.

It is necessary to stress also that a realistic model of a true stock market should include also many other mechanisms to take into account other features like short selling. But this surely requires a better starting point than the model discussed here which, however, we believe is useful to start understanding some basic features and some consequences of our approach.

We end the paper with few final comments. The first is a physical one: people with a quantum mechanical background may be surprised that incompatible (i.e. not commuting) observables appear in the market, and may wonder about their economical meaning. This problem, however, is only apparent since all the observables we are interested in form a commuting subset of a larger non-abelian algebra. Therefore they can be diagonalized simultaneously and a common orthonormal basis of eigenstates can be indeed obtained as in (2.5). The second comment is due to the fact that our figures show continuous plots while our starting assertion was that in the stock market we want to describe only discrete quantities play a role. This is just a matter of interpretation: the continuous lines that we find solving the differential equations are simply the ones which interpolate between the real discrete values which are attained at some particular values of the time which we could interpret as the time for the transaction. Finally, it is clear that another missing aspect of the analysis proposed so far is a comparison with the already existing methods. However, this comparison will be postponed until we will not produce a sufficiently 
complete and realistic model.

\section{Acknowledgements}

This work has been financially supported in part by M.U.R.S.T., within the project Problemi Matematici Non Lineari di Propagazione e Stabilità nei Modelli del Continuo, coordinated by Prof. T. Ruggeri.

\section{Appendix : Few results on the number representation}

We discuss here few important facts in quantum mechanics and second quantization, paying not much attention to mathematical problems arising from the fact that the operators involved are quite often unbounded. More details can be found, for instance, in [10, 11] and [12, as well as in [1, 2].

Let $\mathcal{H}$ be an Hilbert space and $B(\mathcal{H})$ the set of all the bounded operators on $\mathcal{H} . B(\mathcal{H})$ is a $\mathrm{C}^{*}$-algebra, that is an algebra with involution which is complete under a norm $\|$. $\|$ satisfying the so-called $\mathrm{C}^{*}$-property: $\left\|A^{*} A\right\|=\|A\|^{2}$, for all $A \in B(\mathcal{H})$. As a matter of fact $B(\mathcal{H})$ is usually seen as a concrete realization of an abstract $\mathrm{C}^{*}$-algebra. Let $\mathcal{S}$ be our physical system and $\mathfrak{A}$ the set of all the operators useful for a complete description of $\mathcal{S}$, which includes the observables of $\mathcal{S}$. For simplicity it is convenient to assume that $\mathfrak{A}$ is also a $\mathrm{C}^{*}$-algebra, possibly coinciding with $B(\mathcal{H})$ itself, even if this is not always possible. This aspect, related with the importance of some unbounded operators within our scheme, will not be considered here. The description of the time evolution of $\mathcal{S}$ is related to a self-adjoint operator $H=H^{\dagger}$ which is called the hamiltonian of $\mathcal{S}$ and which in standard quantum mechanics represents the energy of $\mathcal{S}$. We will adopt here the so-

called Heisenberg representation, in which the time evolution of an observable $X \in \mathfrak{A}$ is given by

$$
X(t)=e^{i H t} X e^{-i H t}
$$


or, equivalently, by the solution of the differential equation

$$
\frac{d X(t)}{d t}=i e^{i H t}[H, X] e^{-i H t}=i[H, X(t)]
$$

where $[A, B]:=A B-B A$ is the commutator between $A$ and $B$. The time evolution defined in this way is usually a one parameter group of automorphisms of $\mathfrak{A}$.

In our paper a special role is played by the so called canonical commutation relations (CCR): we say that a set of operators $\left\{a_{l}, a_{l}^{\dagger}, l=1,2, \ldots, L\right\}$ satisfy the CCR if the following hold:

$$
\left[a_{l}, a_{n}^{\dagger}\right]=\delta_{l n} \mathbb{1}, \quad\left[a_{l}, a_{n}\right]=\left[a_{l}^{\dagger}, a_{n}^{\dagger}\right]=0
$$

for all $l, n=1,2, \ldots, L$. Here $\mathbb{1}$ is the identity operator. These operators, which are widely analyzed in any textbook in quantum mechanics, see [10] for instance, are those which are used to describe $L$ different modes of bosons. From these operators we can construct $\hat{n}_{l}=a_{l}^{\dagger} a_{l}$ and $\hat{N}=\sum_{l=1}^{L} \hat{n}_{l}$ which are both self-adjoint. In particular $\hat{n}_{l}$ is the number operator for the l-th mode, while $\hat{N}$ is the number operator of $\mathcal{S}$.

The Hilbert space of our system is constructed as follows: we introduce the vacuum of the theory, that is a vector $\varphi_{0}$ which is annihilated by all the operators $a_{l}: a_{l} \varphi_{0}=0$ for all $l=1,2, \ldots, L$. Then we act on $\varphi_{0}$ with the operators $a_{l}^{\dagger}$ and their powers:

$$
\varphi_{n_{1}, n_{2}, \ldots, n_{L}}:=\frac{1}{\sqrt{n_{1} ! n_{2} ! \ldots n_{L} !}}\left(a_{1}^{\dagger}\right)^{n_{1}}\left(a_{2}^{\dagger}\right)^{n_{2}} \cdots\left(a_{L}^{\dagger}\right)^{n_{L}} \varphi_{0}
$$

$n_{l}=0,1,2, \ldots$ for all $l$. These vectors form an orthonormal set and are eigenstates of both $\hat{n}_{l}$ and $\hat{N}: \hat{n}_{l} \varphi_{n_{1}, n_{2}, \ldots, n_{L}}=n_{l} \varphi_{n_{1}, n_{2}, \ldots, n_{L}}$ and $\hat{N} \varphi_{n_{1}, n_{2}, \ldots, n_{L}}=N \varphi_{n_{1}, n_{2}, \ldots, n_{L}}$, where $N=$ $\sum_{l=1}^{L} n_{l}$. Moreover using the CCR we deduce that $\hat{n}_{l}\left(a_{l} \varphi_{n_{1}, n_{2}, \ldots, n_{L}}\right)=\left(n_{l}-1\right)\left(a_{l} \varphi_{n_{1}, n_{2}, \ldots, n_{L}}\right)$ and $\hat{n}_{l}\left(a_{l}^{\dagger} \varphi_{n_{1}, n_{2}, \ldots, n_{L}}\right)=\left(n_{l}+1\right)\left(a_{l}^{\dagger} \varphi_{n_{1}, n_{2}, \ldots, n_{L}}\right)$, for all $l$. For these reasons the following interpretation is given: if the $L$ different modes of bosons of $\mathcal{S}$ are described by the vector $\varphi_{n_{1}, n_{2}, \ldots, n_{L}}$, this implies that $n_{1}$ bosons are in the first mode, $n_{2}$ in the second mode, and so on. The operator $\hat{n}_{l}$ acts on $\varphi_{n_{1}, n_{2}, \ldots, n_{L}}$ and returns $n_{l}$, which is exactly the number of bosons in the l-th mode. The operator $\hat{N}$ counts the total number of bosons. Moreover, the operator $a_{l}$ destroys a boson in the l-th mode, while $a_{l}^{\dagger}$ creates a boson in the same mode. This is why $a_{l}$ and $a_{l}^{\dagger}$ are usually called the annihilation and the creation operators.

The Hilbert space $\mathcal{H}$ is obtained by taking the closure of the linear span of all these vectors. 
An operator $Z \in \mathfrak{A}$ is a constant of motion if it commutes with $H$. Indeed in this case equation (A.2) implies that $\dot{Z}(t)=0$, so that $Z(t)=Z$ for all $t$.

The vector $\varphi_{n_{1}, n_{2}, \ldots, n_{L}}$ in (A.4) defines a vector (or number) state over the algebra $\mathfrak{A}$ as

$$
\omega_{n_{1}, n_{2}, \ldots, n_{L}}(X)=\left\langle\varphi_{n_{1}, n_{2}, \ldots, n_{L}}, X \varphi_{n_{1}, n_{2}, \ldots, n_{L}}\right\rangle,
$$

where $\langle$,$\rangle is the scalar product in the Hilbert space \mathcal{H}$. As we have discussed in [1, 2], these states are used to project from quantum to classical dynamics and to fix the initial conditions of the market.

\section{References}

[1] F. Bagarello, An operatorial approach to stock markets, J. Phys. A, 39, 6823 (2006)

[2] F. Bagarello, Stock Markets and Quantum Dynamics: A Second Quantized Description, Physica A, 386, 283 (2007)

[3] B.E. Baaquie, Quantum Finance, Cambridge University Press (2004)

[4] D. Challet, M. Marsili, Phase transition and symmetry breaking in the minority game, Phis. Rev. E, 60, 6271 (2000)

[5] M. Schaden, A Quantum Approach to Stock Price Fluctuations, Physica A 316, 511 (2002)

[6] R.M. Mantegna, E. Stanley, Introduction to Econophysics, Cambridge University Press (1999)

[7] L. Accardi, Y.G. Lu, I. Volovich, Quantum Theory and its Stochastic Limit, Springer, Berlin (2002)

[8] Lux T., Marchesi M., Scaling and criticality in a stochastic multi-agent model of a financial market, Nature, 397, 498 (1999)

[9] Alfi V., De Martino A., Pietronero L., Tedeschi A., Detecting the traders' strategies in minority-majority games and real stock-prices, Physica A 382, 1 (2007) 
[10] E. Merzbacher, Quantum Mechanics, Wiley, New York (1970),

[11] M.Reed and B.Simon, Methods of Modern Mathematical Physics, I, Academic Press, New York (1980)

[12] O.Bratteli and D.W.Robinson, Operator Algebras and Quantum Statistical Mechanics, I, Springer Verlag, New York, 1979. 